\title{
Equinodermos de Nicaragua: nuevos registros del Pacífico y Caribe Sur
}

Osmar Benito Sandinoํㅡ, Francisco Alonso Solís-Marín², Andrea Alejandra Caballero-Ochoa ${ }^{3}$, Carlos Andrés Conejeros-Vargas ${ }^{1}$, Francisco Márquez-Borrás ${ }^{1}$,

Alejandra Penélope Ayala-Aguilera ${ }^{3}$ \& Alfredo Laguarda-Figueras ${ }^{2}$

1. Posgrado en Ciencias del Mar y Limnología, Instituto de Ciencias del Mar y Limnología (ICML), Universidad Nacional Autónoma de México (UNAM), Apdo. Post. 70-305, Ciudad de México, México, 04510; sandino.lucas08@gmail.com; conejeros@ciencias.unam.mx; marquez@ciencias.unam.mx

2. Laboratorio de Sistemática y Ecología de Equinodermos. Instituto de Ciencias del Mar y Limnología, Universidad Nacional Autónoma de México. Apdo. Post. 70-305, Ciudad de México, México, 04510; fasolis@icml.unam.mx; laguarda@cmarl.unam.mx

3. Facultad de Ciencias, UNAM. Apdo. Post. 70-305, Ciudad de México, México, 04510; penelo92@hotmail.com; a.caballero.ochoa@gmail.com

Recibido 13-I-2017. Corregido 17-V-2017. Aceptado 15-VII-2017.

\begin{abstract}
Echinoderms from Nicaragua: new records for the Pacific and South Caribbean. There is little information about the echinoderms of Nicaragua. Here we present a list based on bibliographical revisions and collections of specimens made in the last three years. Samplings were made from June 11 to 16, 2016, in 10 locations (two in the Pacific and eight in the Caribbean). In addition, the databases of eight international scientific collections were reviewed. Finally, reports were added from photographic archives of the school of diving Dive Nicaragua and ECOLÓGICA S.A. The list of echinoderms of Nicaragua consists of 193 species distributed in five classes, 112 genera, 58 families and 24 orders. The class with the highest number of species was Asteroidea ( 57 species), followed by the class Echinoidea (46 species), and the class with the lowest number of species was Crinoidea (10 species). Fifty six new records are added for Nicaragua, 19 of which correspond to the Pacific and 38 to the Caribbean. Rev. Biol. Trop. 65(Suppl. 1): S288-S298. Epub 2017 November 01.
\end{abstract}

Key words: Echinodermata; Checklist; Corn Island; Little Corn Island; Central America.

El estudio enfocado hacia la biodiversidad marina de Nicaragua dista mucho de estar completo. Aunque es bien conocida la importancia ecológica de los equinodermos dentro de las comunidades marinas, el mar territorial nicaragüense no cuenta con información suficiente que permita describir la diversidad y el papel ecológico de este grupo en las aguas del istmo de Centro América (Quinn-Lampson, Benito-Sandino, Solís-Marín, Arriaga-Ochoa, \& Laguarda-Figueras, 2015).

Los primeros reportes de equinodermos para Nicaragua fueron los realizados por Lyman (1865), quien reportó tres especies de ofiuroideos para la costa oeste de Nicaragua (Ophiactis simplex, Ophiothrix spiculata y
Ophiocoma aethiops); setenta y cinco años más tarde, H.L. Clark (1940) reportó tres especies más de ofiuroideos (Ophiactis savignyi, Ophioderma teres y $O$. panamensis) para la misma costa. Así mismo, varias especies de equinodermos han sido descritas usando sitios de las costas nicaragüenses como localidades tipo, tal es el caso de Ophiolepis grisea H.L. Clark, 1940 (Manypenny Point, Golfo de Fonseca); Ophioderma teres var. unicolor H.L. Clark, 1940 (Isla Cardón, Corinto) y Encope laevis H.L. Clark, 1948 (noroeste de Corinto).

Los antecedentes de estudios sobre equinodermos de Centroamérica corresponden a revisiones de las colecciones más importantes en México y Estados Unidos (Alvarado, 
Solís-Marín, \& Ahearn, 2008; Alvarado et al., 2011; Alvarado, 2011; Alvarado \& SolísMarín, 2013). Hasta hace un par de años, el resultado de los trabajos citados anteriormente enumeraban 137 especies de equinodermos reportados para las costas de Nicaragua (Quinn-Lampson et al., 2015).

Este estudio representa también la realización de muestreos más sistemáticos en ciertas localidades donde no se habían hecho trabajos de este tipo, tanto para el Pacífico como para el Caribe nicaragüense.

El objetivo de este trabajo es presentar el listado taxonómico actualizado de los equinodermos de Nicaragua, resultado de las revisiones bibliográficas, así como de las recolectas puntuales hechas en los últimos tres años.

\section{MATERIALES Y MÉTODOS}

Para la realización de este trabajo, se llevaron a cabo muestreos en la costa del Pacífico de Nicaragua, en San Juan del Sur (SJdS, $\left.11^{\circ} 23^{\prime} \mathrm{N}, 86^{\circ} 02^{\prime} \mathrm{W}\right)$ y en el Caribe, en Corn Island $\left(12^{\circ} 10^{\prime} 00^{\prime} \mathrm{N}, 83^{\circ} 02^{\prime} 00^{\prime} \mathrm{W}\right)$. Según Cortés (2007) y Alvarado et al. (2011) la bahía de San Juan del Sur se caracteriza por ser una zona rocosa e influenciada por los fenómenos de surgencias. Cortés (2007) reporta además, pequeñas colonias coralinas (Pocillopora spp.) en la parte más norteña de la bahía, las cuales se vuelven más abundantes a medida que nos acercamos a las costas costarricenses. Corn Island pertenece al municipio de la Región Autónoma de la Costa Caribe Sur (RACS) conformado por dos islas; Great y Little Corn Island, ambas presentan sistemas de arrecifes bien conservados (Ryan \& Zapata, 2003). Las zonas caribeñas estudiadas presentan una mayor diversidad de sustratos (arena, ostrales, parches coralinos, parches de octocoral, praderas de fanerógamas: Thalassia testudinum y Syringodium filiforme) que permite la presencia de una mayor cantidad de especies de equinodermos (Ryan \& Zapata, 2003) (Figs. 1 y 2).

Revisión de material: Para la obtención de los registros previos de ejemplares de las especies de equinodermos recolectadas en Nicaragua, se consultaron las bases de datos de las siguientes colecciones: Colección Nacional de Equinodermos "Dra. María Elena Caso Muñoz", Instituto de Ciencias del Mar y Limnología, UNAM, Ciudad de México, México (CNE); California Academy of Sciences, San Francisco, California, U.S.A. (CAS); Museum of Comparative Zoology, Harvard University, U.S.A. (MCZ); United National Museum of Natural History, Smithsonian Institution, Washington, D.C., U.S.A. (USNM); Biodiversity Research and Teaching Collections, Department of Wildlife and Fisheries Sciences, Texas A\&M University, College Station, TX, U.S.A. (TCWC); Invertebrate Zoology, Florida Museum of Natural History, Florida, U.S.A. (UF); National History Museum at South Kensington, London (NHMUK) y Australian Museum provider for OZCAM, Collection of Invertebrates, Marine \& Other, Australia (AM). Los ejemplares de las colecciones CNE, CAS, MCZ y USNM fueron revisados por FASM (coautor de este trabajo) en visitas previas. A su vez, se anexaron en el presente listado los registros de las especies reportadas por Alvarado, Solis-Marin, \& Ahearn (2010) y por Alvarado et al. (2008, 2011, 2013), Solís-Marín et al. (2013) y Quinn-Lampson et al. (2015). Además, se revisaron los archivos fotográficos compartidos por Fabio Buitrago Vannini y Edgar Castañeda (escuela de buceo Dive Nicaragua y ECOLÓGICA S.A.), para que, previa identificación de las especies, se incluyeran los registros en el presente trabajo.

Trabajo de campo: La fase de campo se desarrolló del 11 al 16 de junio 2016. La recolección de ejemplares se realizó utilizando diferentes métodos: buceo libre (en el Pacífico) y buceo autónomo (en el Caribe), las profundidades de recolecta variaron de 0 a $8.5 \mathrm{~m}$. Los muestreos se realizaron en diez localidades, dos en el Pacífico (frente a la Talanguera, San Juan del Sur y Playa Marsella, Marsella) y ocho en el Caribe (cinco localidades en Corn Island: La Virgen, Frente a NACO, Woula Point, Frente a Spekito y La Princesa; y tres localidades en 

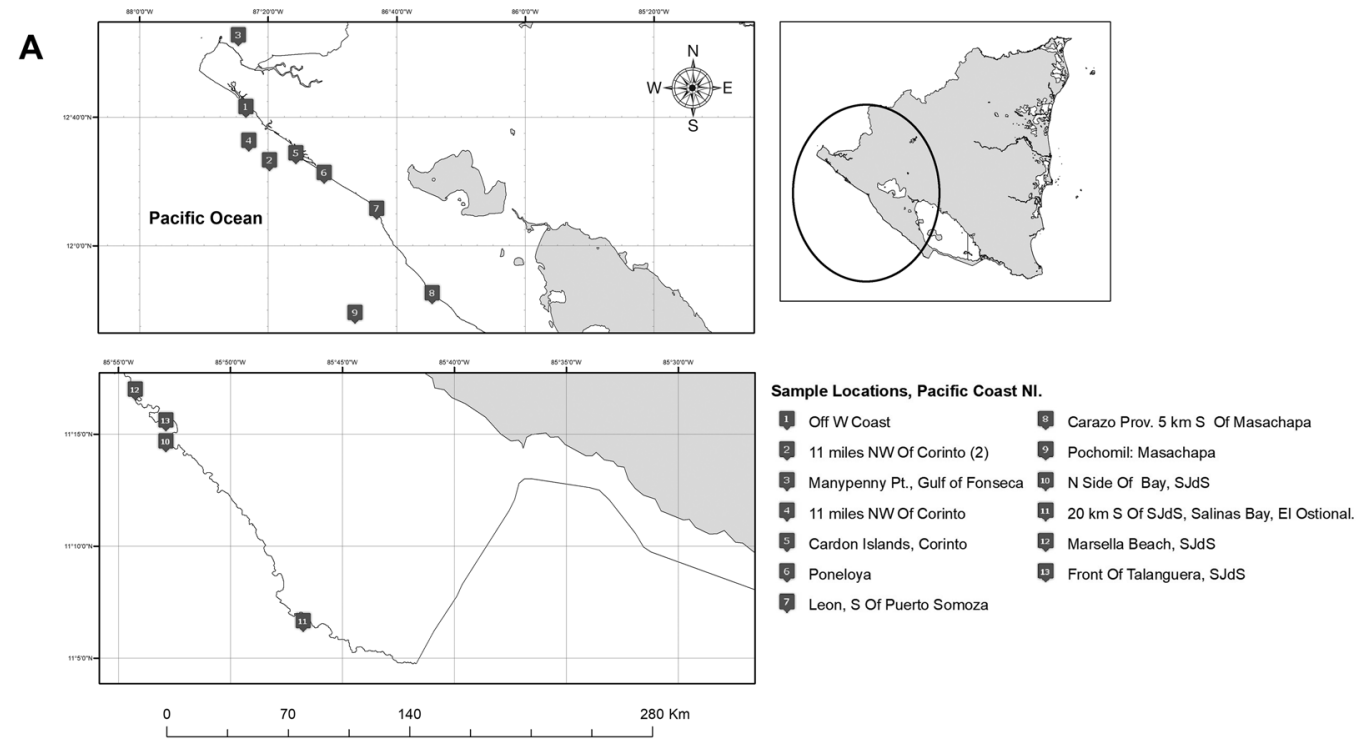

7 Leon, S Of Puerto Somoza
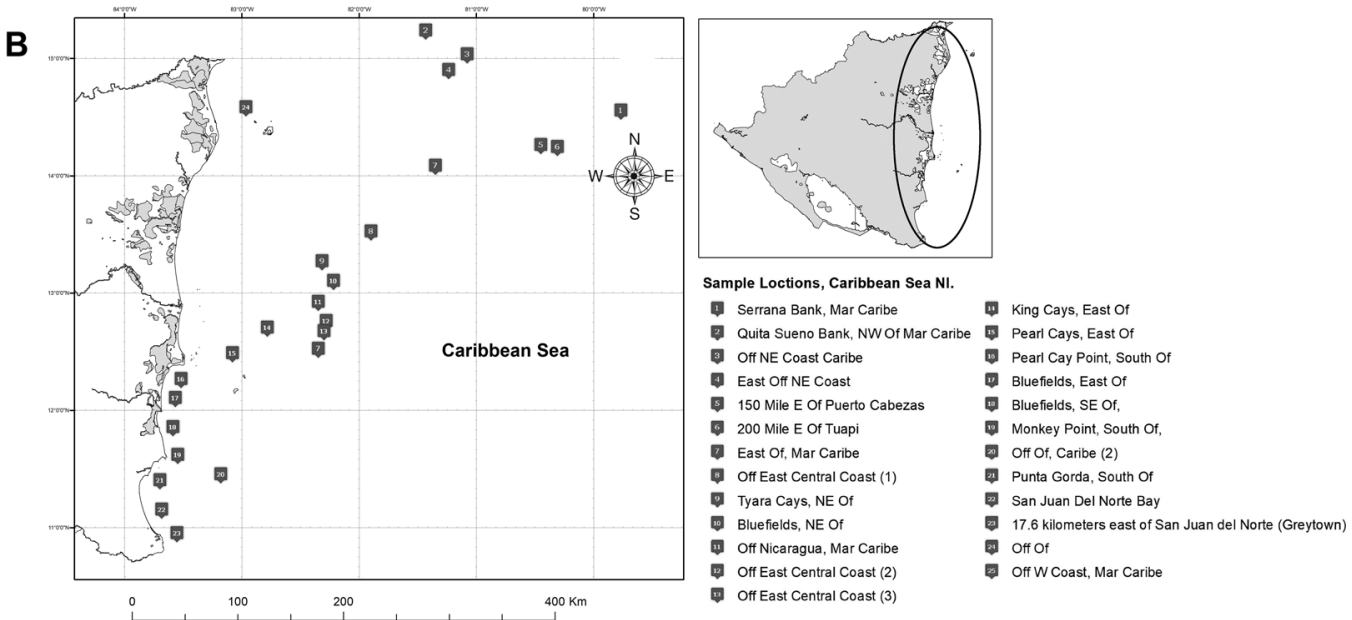

Fig. 1. A. Sitios de muestreo de equinodermos en la costa del Pacífico nicaragüense, (sitios 1-2 corresponden a registros obtenidos del USNM; sitios 3-5 corresponden a registros obtenidos de la base de datos del MCZ; sitios 6-11 corresponden a registros obtenidos de la base de datos del CAS; sitios 12-13 corresponden a registros obtenidos del muestreo del presente estudio). B. Sitios de muestreo de equinodermos en la costa Caribe y mar profundo nicaragüense, (sitios 1-24 corresponden a registros obtenidos del USNM).

Fig. 1. A. Echinoderm sampling sites on the Pacific coast of Nicaragua (sites 1-2 corresponds to records obtained from the USNM; sites 3-5 correspond to records obtained from the MCZ database; sites 6-11 correspond to records obtained from CAS database; sites 12-13 correspond to records obtained from the sampling of the present study). B. Echinoderm sampling sites on the Caribbean coast and deep sea of Nicaragua, (sites 1-24 correspond to records obtained from the USNM).

Little Corn Island: South West, Dolphin Dive Point y LCI-3) (Figs. 1 y 2). Dichas localidades se seleccionaron tomando en cuenta el conocimiento previo de las áreas cercanas a algunos arrecifes importantes, buscando abarcar la mayor cantidad de sustratos (arenales, coral vivo, coral muerto, esponjas, rocas grandes, praderas de pastos marinos y mantos algales).

Una vez recolectados y seleccionados los ejemplares que serían preservados, éstos fueron 
A

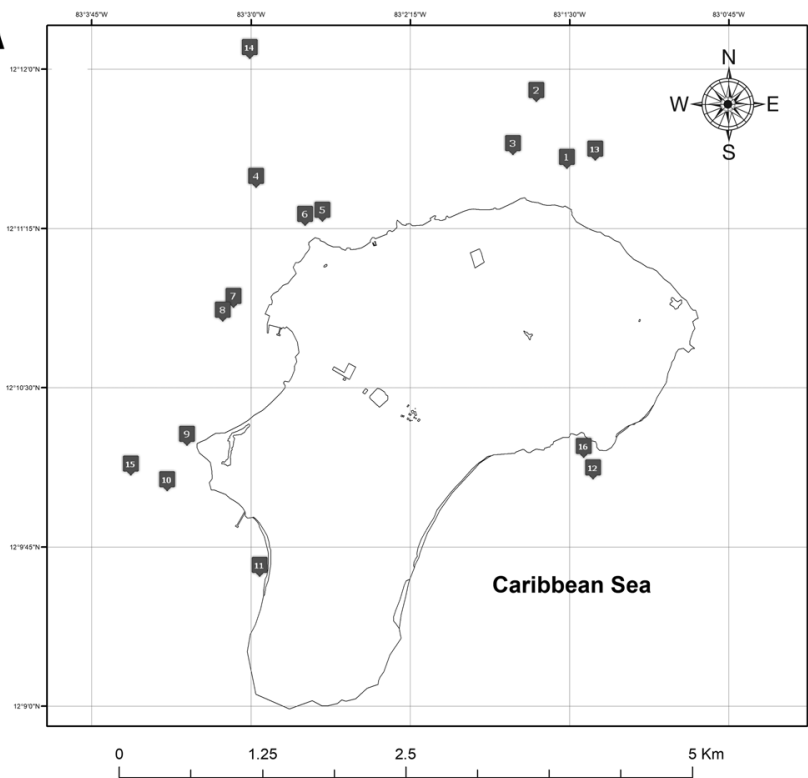

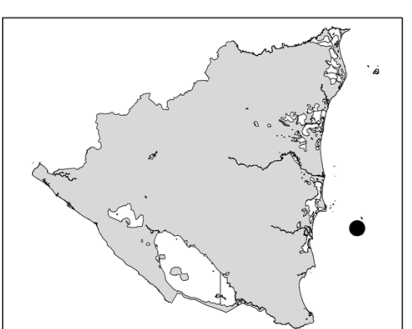

Sample Locations, Corn Island NI.

4. Sandfly Bay

q La Princesa

2. Iglesia Bautista

3. Fisherman Union

(10) Brad Bay

4. Front Road

4. Picnic Center

5. Kaiko Hote

1. Bambule Point

6. Anastasia

7. Acopio Harry

8. Dock Point

NACO

(14) La Virgen

15 Woula Point

5pekito
B

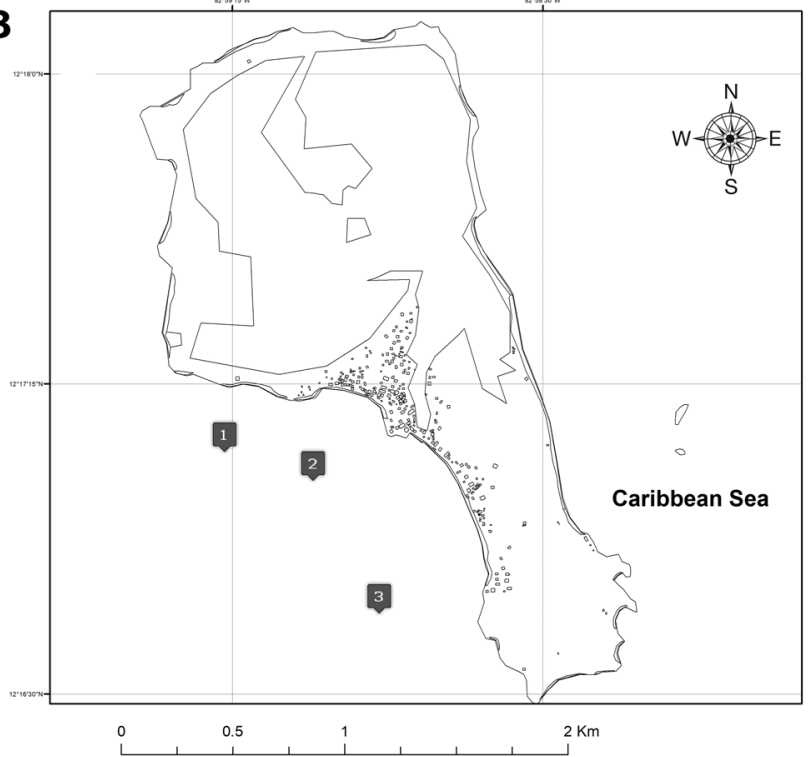

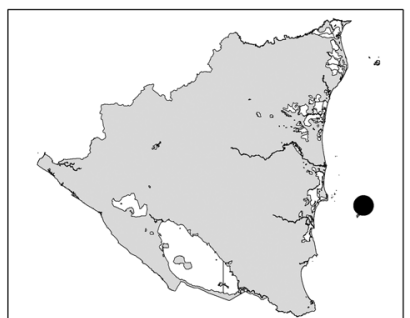

Sample Locations, Little Corn Island NI.

1 South West, $\mathrm{LCl}$

2 Dolphin Dive, $\mathrm{LCl}$

$3 . \mathrm{LCl}-3$

Fig. 2. A. Sitios de muestreo de equinodermos en Big Corn Island, Caribe nicaragüense (sitios 1-12 corresponden a registros obtenidos de Quinn-Lampson, Benito-Sandino, Solís-Marín, Arriaga-Ochoa, \& Laguarda-Figueras [2015]); sitios 13-16 corresponden a registros obtenidos del muestreo del presente estudio. B. Sitios de muestreo de equinodermos en Little Corn Island, Caribe nicaragüense, (sitios 1-3 corresponden a registros obtenidos del muestreo del presente estudio).

Fig. 2. A. Echinoderm sampling sites in Big Corn Island, Nicaraguan Caribbean (sites 1-12 correspond to records obtained from Quinn-Lampson, Benito-Sandino, Solís-Marín, Arriaga-Ochoa, \& Laguarda-Figueras [2015]), sites 13-16 correspond to records obtained from the sampling in the present study. B. Echinoderms sampling sites in Little Corn Island, Nicaraguan Caribbean (sites 1-3 correspond to records obtained from sampling in the present study). 
relajados con una solución de agua de mar y cloruro de magnesio $\left(\mathrm{MgCl}_{2}\right)$. Ya narcotizados los ejemplares fueron preservados en alcohol etílico al 70\% (Hendler, Miller, Pawson, \& Kier, 1995). La revisión taxonómica y la identificación de los ejemplares fue realizada en las instalaciones de la Bluefields Indian \& Caribbean University (BICU), Corn Island y en el Laboratorio de Sistemática y Ecología de Equinodermos (LSEE), Instituto de Ciencias del Mar y Limnología (ICML), Universidad Nacional Autónoma de México (UNAM), Ciudad de México, México. Los ejemplares recolectados fueron depositados en la Colección de Zoología de Invertebrados, Departamento de Biología, Universidad de León, Universidad Nacional Autónoma de Nicaragua (UNANLeón), Nicaragua, y en la Colección Nacional de Equinodermos (CNE) "Dra. María Elena Caso Muñoz" del ICML, UNAM, México.

\section{RESULTADOS}

Durante el trabajo de campo realizado se recolectaron 135 ejemplares, pertenecientes a 74 especies de equinodermos, distribuidas de la siguiente manera: Asteroidea (nueve especies), Ophiuroidea (31 especies), Echinoidea (13 especies) y Holothuroidea (21 especies); 23 de estas especies fueron nuevos registros para
Nicaragua. Con respecto a la costa del Pacífico se recolectaron 45 especímenes, repartidos en 23 especies (Asteroidea una, Ophiuroidea nueve, Holothuroidea 12 y Echinoidea tres) y en el Caribe se recolectaron 90 especímenes, agrupados en 50 especies (Asteroidea ocho, Ophiuroidea 24, Holothuroidea ocho y Echinoidea diez).

El listado actualizado de equinodermos de Nicaragua está conformado por 193 especies distribuidas en cinco clases, 24 órdenes, 58 familias y 112 géneros (Cuadro 1, 2). La clase con el mayor número de especies fue Asteroidea (57 especies), seguida por la clase Echinoidea (46 especies), y la clase con menor número de especies fue Crinoidea (diez especies). La familia mejor representada de estrellas de mar en Nicaragua es la Astropectinidae, representada por 12 especies de paxilósidos, seguida por la familia Goniasteridae con nueve especies. La familia de ofiuroideos mejor representada en aguas nicaragüenses es la familia Ophiodermatidae con 11 especies, seguida por la familia Ophiothrichidae, formada por un grupo de siete especies. La familia mejor representada de erizos de mar en aguas de Nicaragua es la familia Mellitidae representada por siete especies. La familia de pepinos de mar mejor representada en Nicaragua es la familia Holothuriidae con 15 especies, casi todas ellas del género Holothuria.

CUADRO 1

Riqueza de equinodermos de Nicaragua por clase y sus categorías taxonómicas descendentes

TABLE 1

Echinoderm richness of Nicaragua by class and their descending taxonomic categories

\begin{tabular}{lcccc} 
& $\begin{array}{c}\text { Órdenes } \\
\text { Pacífico/Caribe }\end{array}$ & $\begin{array}{c}\text { Familias } \\
\text { Pacífico/Caribe }\end{array}$ & $\begin{array}{c}\text { Géneros } \\
\text { Pacífico/Caribe }\end{array}$ & $\begin{array}{c}\text { Especies } \\
\text { Pacífico/Caribe }\end{array}$ \\
Crinoidea & $0 / 2$ & $0 / 4$ & $0 / 7$ & $0 / 10$ \\
Asteroidea & $4 / 6$ & $7 / 13$ & $9 / 30$ & $10 / 47$ \\
Ophiuroidea & $1 / 2$ & $7 / 10$ & $9 / 15$ & $13 / 29$ \\
Echinoidea & $6 / 10$ & $8 / 18$ & $13 / 26$ & $15 / 31$ \\
Holothuroidea & $3 / 5$ & $7 / 10$ & $10 / 15$ & $20 / 21$ \\
Total & $\mathbf{1 4 / 2 5}$ & $29 / 55$ & $41 / 93$ & $58 / 138^{*}$ \\
\hline
\end{tabular}

*La sumatoria del número total de registros de especies para el país (194), no concuerda con los números reales (número de especies), ya que existen tres especies de equinodermos (Ophiactis savignyi, Holothuria arenicola y H. impatiens) que habitan tanto el Pacífico como el Caribe nicaragüense. 
El Pacífico nicaragüense cuenta con 58 registros de especies de equinodermos, conformados por 10 especies de la clase Asteroidea, 13 especies de la clase Ophiuroidea, 15 especies de la clase Echinoidea y 20 especies de la clase Holothuroidea. El Caribe nicaragüense cuenta con 138 registros de especies de equinodermos, conformados por 10 especies de la clase Crinoidea, 47 especies de la clase Asteroidea, 29 especies de la clase Ophiuroidea, 31 de la clase Echinoidea y 21 especies de la clase Holothuroidea (Cuadro 1).

En el listado taxonómico de los equinodermos de Nicaragua (Cuadro 2) los registros obtenidos del trabajo de Alvarado et al. (2008) se indican con el superíndice 1; los registros de Alvarado et al. (2010) se indican con el superíndice 2; los registros para las especies obtenidas de Alvarado (2011) se indican con el superíndice 3 ; los registros obtenidos de Alvarado et al. (2013) se indican con el superíndice 4; los registros obtenidos de Quinn-Lampson et al. (2015) se indican con el superíndice 5. En el listado también se incluyeron los registros fotográficos de la escuela de buceo Dive Nicaragua y ECOLÓGICA S.A. indicados con el superíndice 6. Finalmente, los nuevos registros están señalados en negritas y por un asterisco $(*)$. Se señalan con dos astericos $(* *)$ los nuevos registros obtenidos en éste trabajo y con tres asteriscos $(* * *)$ los nuevos registros obtenidos a partir de fotografías de la Escuela de Buceo Dive Nicaragua y ECOLÓGICA S. A. Inmediatamente después del nombre científico se añadió la letra " $C$ " para los registros del Caribe nicaragüense, y la letra "P" para los registros del Pacífico nicaragüense.
CUADRO 2

Listado taxonómico de los equinodermos de Nicaragua

TABLE 2

Taxonomic list of the echinoderms of Nicaragua

El arreglo taxonómico del presente listado está basado en el Catálogo de Autoridades Taxonómicas de los Equinodermos de México (Solís-Marín, Caballero-Ochoa, FrontanaUribe, Laguarda-Figueras, \& Durán-González, 2017)

The taxonomic arrangement of this taxonomic list is based on the Catalog of Taxonomic Authorities of Echinoderms of Mexico (Solís-Marín, Caballero-Ochoa, FrontanaUribe, Laguarda-Figueras, \& Durán-González, 2017)

\section{CLASE CRINOIDEA Miller, 1821}

Orden Comatulida A.H. Clark, 1908

Familia Antedonidae Norman, 1865 Trichometra cubensis (Pourtalès, 1869) ${ }^{1}$ : C; USNM

Familia Bourgueticrinidae de Loriol, 1882 Democrinus rawsoni (Pourtalès, 1874) ${ }^{1}$ : C; USNM Democrinus conifer (A.H. Clark, 1909) ${ }^{1}$ : C; USNM

Familia Comatulidae Fleming, 1828 Comactinia echinoptera (J. Müller, 1840) ${ }^{1}$ : C; USNM Comactinia meridionalis meridionalis (L. Agassiz, 1865) ${ }^{1}$ : C; USNM

Davidaster discoideus (Carpenter, 1888) ${ }^{1}$ : C Nemaster grandis A.H. Clark, $1909^{1}$ : C; USNM Neocomatella alata (Pourtalès, 1878)*: C; UF Neocomatella pulchella (Pourtalès, 1878) ${ }^{1}$ : C; USNM

Familia Charitometridae A.H. Clark, 1909 Crinometra brevipinna (Pourtalès, 1868) ${ }^{1}$ : C; USNM

Orden Millericrinida Sieverts-Doreck, 1952

CLASE ASTEROIDEA de Blainville, 1830

Orden Forcipulatida Perrier, 1884

Familia Heliasteridae Viguier, 1878 Heliaster cumingi (Gray, 1840) ${ }^{2}$ : P

Familia Pedicellasteridae Perrier, 1884 Pedicellaster pourtalesi Perrier, $1881^{3}$ : C; NHMUK

Familia Zoroasteridae Sladen, 1889 Zoroaster fulgens Wyville-Thomson, 1873 *: C; TCWC

Orden Notomyotida Ludwig, 1910

Familia Benthopectinidae Verrill, 1899 Benthopecten simplex (Perrier, 1881) *: C; TCWC Cheiraster (Barbadosaster) echinulatus (Perrier, 1875) ${ }^{1}$ : C; USNM

Cheiraster (Christopheraster) mirabilis (Perrier, 1881) ${ }^{1}$ : C; USNM

Orden Paxillosida Perrier, 1884

Familia Astropectinidae Gray, 1840 Astropecten alligator Perrier, $1881^{1}{ }^{1}$ : C; USNM Astropecten americanus (Verrill, 1880) ${ }^{1}$ : C; USNM Astropecten articulatus (Say, 1825) ${ }^{1}$ : C; USNM Astropecten cingulatus Sladen, $1883^{1}$ : C; USNM Astropecten comptus Verrill, 1915 *: C; TCWC 
Astropecten duplicatus Gray, $1840^{1}$ : C; USNM

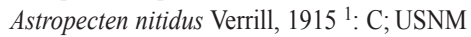

Blakiaster conicus Perrier, 1881 *: C; NHMUK

Dytaster insignis (Perrier, 1884) *: C; TCWC

Persephonaster echinulatus H.L. Clark, $1941^{1}$ : C; USNM

Persephonaster patagiatus (Sladen, 1889) ${ }^{3}$ : C

Tethyaster grandis (Verrill, 1899) *: C; USNM

Familia Goniopectinidae Verrill, 1899

Prionaster elegans Verrill, $1899^{1}$ : C; USNM

Familia Luidiidae Sladen, 1889

Luidia barbadensis Perrier, $1881^{1}$ : C; USNM

Luidia clathrata (Say, 1825) ${ }^{1}$ : C; USNM

Luidia foliolata Grube, $1866^{2}$ : $\mathrm{P}$

Luidia (Platasterias) latiradiata (Gray, 1871) ${ }^{2}$.

P; USNM, CAL

Luidia lawrencei Hopkins \& Knott, $2010 * *$ : C

Luidia senegalensis (Lamarck, 1816) ${ }^{1}$ : C; USNM

Orden Spinulosida Perrier, 1884

Familia Echinasteridae Verrill, 1870

Echinaster (Echinaster) modestus Perrier, $1881^{1}$ : C

Echinaster (Othilia) aculeata (Gray, 1840) 2: P

Echinaster (Othilia) brasiliensis Müller \& Troschel, 1842*: C; USNM

Echinaster (Othilia) echinophorus (Lamarck, 1816) ${ }^{3}$ : C

Echinaster (Othilia) guyanensis A.M. Clark, $1987^{1}$ :

C; USNM

Echinaster (Othilia) spinulosus Verrill, $1869^{1}$ : C

Henricia antillarum (Perrier, 1881) ${ }^{1}$ : C; USNM

Orden Valvatida Perrier, 1884

Familia Asterinidae Gray, 1840

Asterinides folium (Lütken, 1859) **: C

Asterinides pompom (A.M. Clark, 1983) **: C

Familia Goniasteridae Forbes, 1841

Anthenoides piercei Perrier, $1881^{1}$ : C; USNM

Ceramaster grenadensis grenadensis (Perrier, 1881) ${ }^{1}$.

C; USNM

Ceramaster patagonicus (Sladen, 1889) *: P; CAS

Circeaster americanus (A.H. Clark, 1916) ${ }^{1}$ : C; USNM

Litonotaster intermedius (Perrier, 1884) *: C; TCWC

Nymphaster arenatus (Perrier, 1881) ${ }^{1}$ : C; USNM

Plinthaster dentatus (Perrier, 1884) ${ }^{1}$ : C; USNM

Pseudarchaster gracilis gracilis (Sladen, 1889) ${ }^{1}$ : C; USNM

Rosaster alexandri (Perrier, 1881) ${ }^{4}$ : C; USNM

Familia Mithrodiidae Viguier, 1878

Mithrodia bradleyi Verrill, $1870{ }^{6 * * *}$ : P

Mithrodia clavigera (Lamarck, 1816) ${ }^{1}$ : C; USNM

Familia Ophidiasteridae Verrill, 1870

Linckia guildingii Gray, $1840^{5}$ : C

Ophidiaster guildingi Gray, $1840 * *$ : C

Pharia pyramidata (Gray, 1840) ${ }^{6 * * *}$ : P

Phataria unifascialis (Gray, 1840) ${ }^{2}: \mathrm{P}$

Tamaria halperni Downey, 1971 *: C; USNM

Familia Oreasteridae Fisher, 1911

Nidorellia armata (Gray, 1840) ${ }^{2}: \mathrm{P}$

Oreaster reticulatus (Linnaeus, 1758) ${ }^{1}$ : C; USNM

Pentaceraster cumingi (Gray, 1840) ${ }^{6 * * *}$ : P
Orden Velatida Perrier, 1884

Familia Pterasteridae Perrier, 1875

Calyptraster personatus (Perrier, 1885) *: C; TCWC

Hymenaster modestus Verrill, 1885 *: C; TCWC

Pteraster militarioides militarioides H.L. Clark, $1941^{1}{ }^{~}$ :

C; USNM

Pteraster militaris (O.F. Müller, 1776) ${ }^{4}$ : C

\section{CLASE OPHIUROIDEA Gray, 1840}

Orden Euryalida Lamarck, 1816

Familia Gorgonocephalidae Ljungman, 1867 Astrophyton muricatum (Lamarck, 1816) ${ }^{1}$ : C

Orden Ophiurida Müller \& Troschel, 1840

Familia Ophiomyxidae Ljungman, 1867 Ophiomyxa flaccida (Say, 1825) ${ }^{5}$ : C

Familia Amphiuridae Ljungman, 1867 Amphiodia pulchella (Lyman, 1869) **: C Amphiodia sculptilis Ziesenhenne, $1940^{2}$ : P Amphipholis squamata (Delle Chiaje, 1828) ${ }^{2}$ : P

Amphiura palmeri Lyman, $1882 * *$ : C

Ophiophragmus ophiactoides Ziesenhenne, 1940 *: C; AM

Familia Ophiactidae Matsumoto, 1915

Hemipholis gracilis Verrill, $1867^{2}$ : $\mathrm{P}$

Ophiactis muelleri Lütken, $1856 * *$ : C

Ophiactis savignyi (Müller \& Troschel, 1842): C, $\mathrm{P}^{4}$;

USNM *Nuevo registro para el Caribe nicaragüense

Ophiactis simplex (Le Conte, 1851) ${ }^{2}$ : P

Familia Ophiothricidae Ljungman, 1867 Ophiothrix angulata (Say, 1825) ${ }^{5}$ : C

Ophiothrix cimar Hendler, $2005 * *$ : C

Ophiothrix lineata Lyman, $1860^{5}$ : C

Ophiothrix orstedii Lütken, $1856^{5}$ : C

Ophiothrix pallida Ljugman, $1871^{1}$ : C; USNM

Ophiothrix spiculata Le Conte, $1851^{2}$ : P; CAS, USNM

Ophiothrix suensonii Lütken, $1856^{5}$ : C

Familia Ophiocomidae Ljungman, 1867

Ophiocoma aethiops Lütken, $1859^{2}$ : P; USNM

Ophiocoma alexandri Lyman, $1860^{2}$ : $\mathrm{P}$

Ophiocoma echinata (Lamarck, 1816) ${ }^{1}$ : C

Ophiocoma pumila Lütken, $1856^{5}$ : C

Ophiocoma wendtii Müller \& Troschel, $1842^{5}$ : C

Ophiopsila riisei Lütken, $1859 * *$ : C

Familia Ophionereididae Ljungman, 1867

Ophionereis annulata (Le Conte, 1851) **: P

Ophionereis reticulata (Say, 1825) ${ }^{5}$ : C

Familia Ophiacanthidae Perrier, 1891 Ophiocamax fasciculata Lyman, $1883^{1}$ : C; USNM

Familia Ophiodermatidae Ljungman, 1867

Bathypectinura heros (Lyman, 1879) ${ }^{1}$ : C; USNM

Ophioderma appressa (Say, 1825) ${ }^{5}$ : C

Ophioderma brevicauda Lütken, 1956 **: C

Ophioderma brevispina (Say, 1825) ${ }^{5}$ : C

Ophioderma cinerea Müller \& Troschel, $1842^{5}$ : C

Ophioderma guttata Lütken, $1859^{5}$ : C

Ophioderma panamensis Lütken, $1859^{2}$ : P; MCZ

Ophioderma phoenia H.L. Clark, $1918^{5}$ : C 
Ophioderma rubicunda Lütken, $1856^{5}$ : C Ophioderma teres (Lyman, 1860) 2: P; MCZ Ophioderma variegata Lütken, $1856^{2}$ : P; MCZ

Familia Ophiolepididae Ljungman, 1867 Ophiolepis grisea H.L. Clark, $1940^{2}$ : P; MCZ Ophiolepis impressa Lütken, $1859^{5}$ : C Ophiothyreus goesi Ljungman, $1872^{1}$ : C; USNM

\section{CLASE ECHINOIDEA Leske, 1778}

Orden Cidaroida Claus, 1880

Familia Cidaridae Gray, 1825 Eucidaris thouarsii (L. Agassiz \& Desor, 1846) ${ }^{4}$ : P Eucidaris tribuloides (Lamarck, 1816) ${ }^{1}$ : C; USNM Hesperocidaris asteriscus H.L. Clark, 1948 **: P Stylocidaris affinis (Philippi, 1845) ${ }^{1}$ : C; USNM Stylocidaris lineata Mortensen, $1910^{1}$ : C; USNM

Familia Histocidaridae Lambert, 1900 Poriocidaris purpurata (Wyville-Thomson, 1872) ${ }^{1}$ : C; USNM

Orden Aspidodiatematoida Kroh \& A.B. Smith, 2010

Familia Aspidodiadematidae Duncan, 1889 Aspidodiadema jacobyi A. Agassiz, $1880^{1}$ ': C; USNM

Orden Diadematoida Duncan, 1889

Familia Diadematidae Gray, 1855 Astropyga pulvinata (Lamarck, 1816) ${ }^{4}$ : $\mathrm{P}$ Diadema antillarum (Philippi, 1845) ${ }^{1}$ : C; USNM Diadema mexicanum A. Agassiz, $1863^{4}$ : P

Orden Arbacioida Gregory, 1900

Familia Arbaciidae Gray, 1855 Arbacia stellata (Blainville, 1825) ${ }^{2}$ : P; CAS Coelopleurus floridanus A. Agassiz, 1872 *: C; USNM

Orden Camarodonta Jackson, 1912

Familia Echinometridae Gray, 1855 Echinometra lucunter (Linnaeus, 1758) ${ }^{1}$ : C; USNM Echinometra vanbrunti A. Agassiz, $1863^{2}$ : $\mathrm{P}$ Echinometra viridis A. Agassiz, $1863^{5}$ : C

Familia Toxopneustidae Troschel, 1872 Lytechinus variegatus (Lamarck, 1816) ${ }^{5}$ : C Toxopneustes roseus (A. Agassiz, 1863) ${ }^{4}$ : P Tripneustes depressus A. Agassiz, $1863^{4}$ : $\mathrm{P}$ Tripneustes ventricosus (Lamarck, 1816) ${ }^{5}$ : C

Orden Echinothurioida Claus, 1880

Familia Echinothuriidae Wyville-Thomson, 1872 Araeosoma belli Mortensen, $1903^{1}$ : C; USNM

Familia Phormosomatidae Mortensen, 1934 Phormosoma placenta placenta Wyville-Thomson, $1872^{1}$ : C

Phormosoma placenta sigsbei A. Agassiz, 1880 *: C; USNM

Orden Spatangoida L. Agassiz, 1840

Familia Brissidae Gray, 1855

Brissopsis atlantica Mortensen, $1907^{\text {1: }}$ C; USNM

Brissus unicolor (Leske, 1778) **: C

Meoma ventricosa (Lamarck, 1816) ${ }^{1}$ : C

Plagiobrissus grandis (Gmelin, 1788) ${ }^{5}$ : C

Plethotaenia spatangoides (A. Agassiz, 1883) ${ }^{1}$ : C; USNM
Familia Eurypatagidae Kroh, 2007 Linopneustes longispinus (A. Agassiz, 1878) ${ }^{1}$ : C; USNM

Familia Paleopneustidae A. Agassiz, 1904 Paleopneuestes cristatus A. Agassiz, 1873 *: C; TCWC Heterobrissus hystrix (A. Agassiz, 1880) ${ }^{1}$ : C; USNM

Familia Prenasteridae Lambert, 1905 Agassizia excentrica A. Agassiz, $1869^{1}$ : C Agassizia scrobiculata Valenciennes, $1846^{2}$ : P; CAS

Familia Schizasteridae Lambert, 1905 Schizaster doederleini (Chesher, 1972) ${ }^{1}$ : C; USNM

Orden Echinoneoida H.L. Clark, 1925

Familia Echinoneidae L. Agassiz \& Desor, 1847 Echinoneus cyclostomus Leske, $1778 * *$ : C

Orden Clypeasteroida L. Agassiz, 1872

Familia Clypeasteridae L. Agassiz, 1835 Clypeaster lamprus H.L. Clark, $1914^{1}$ : C; USNM Clypeaster rosaceus (Linnaeus, 1758) ${ }^{5}$ : C Clypeaster rotundus (A. Agassiz, 1863) 22: $\mathrm{P}$ Clypeaster subdepressus (Gray, 1825) ${ }^{5}$ : C

Familia Mellitidae Stefanini, 1912 Encope grandis L. Agassiz, $1841^{2}$ : P Encope laevis H.L. Clark, $1948^{2}$ : P; USNM, MCZ, CAS, CNE

Encope micropora L. Agassiz, $1841^{2}$ : P; USNM Lanthonia longifissa (Michelin, 1858) ${ }^{2}$ : P; CAS Leodia sexiesperforata (Leske, 1778) ${ }^{5}$ : C Mellita notabilis H.L. Clark, $1947^{2}$ : P; CAS Mellita quinquiesperforata (Leske, 1778) ${ }^{1}$ : C; CAS

Orden Echinolampadoida Kroh \& A.B. Smith, 2010

Familia Echinolampadidae Gray, 1851 Conolampas sigsbei (A. Agassiz, 1878) ${ }^{1}$ : C; USNM

CLASE HOLOTHUROIDEA de Blainville, 1834

Orden Dendrochirotida Grube, 1840

Familia Cucumariidae Ludwig, 1894 Cucumaria flamma Solís-Marín \& Laguarda-Figueras, $1999^{4}: \mathrm{P}$ Pseudocnus californicus (Semper, 1868) 2: P Parathyone suspecta (Ludwig, 1875) ${ }^{5}$ : C Neocucumis veleronis (Deichmann, 1941) ${ }^{2}$ : P; USNM

Familia Phyllophoridae Östergren, 1907 Euthyonidiella occidentalis (Ludwig, 1875) ${ }^{5}$ : C Pentamera chierchia (Ludwig, 1887) **: P

Familia Psolidae Burmeister, 1867 Lissothuria ornata Verrill, $1867^{2}$ : P; USNM

Familia Sclerodactylidae Panning, 1949 Neothyone gibber (Selenka, 1867) **: P Neothyone gibbosa Deichmann, 1941 **: P Pachythyone pseudolugubris Deichmann, 1941 **: P Orden Aspidochirotida Grube, 1840

Familia Holothuriidae Ludwig, 1894 Actinopyga agassizi (Selenka, 1867) ${ }^{5}$ : C; Holothuria (Cystipus) cubana Ludwig, $1875 * *$ : C Holothuria (Cystipus) inhabilis Selenka, 1867 *: P; MCZ Holothuria (Halodeima) floridana Pourtalès, $1851^{5}$ : C Holothuria (Halodeima) inornata Semper, $1868 * *$ : P 
Holothuria (Halodeima) kefersteinii (Selenka, 1867) *: P; MCZ

Holothuria (Halodeima) mexicana Ludwig, $1875^{1}$ :

C; USNM

Holothuria (Platyperona) difficilis Semper, 1868 2: P

Holothuria (Selenkothuria) carere Honey-Escandón \& Solís-Marín, 2011 **: P

Holothuria (Selenkothuria) lubrica Selenka, 1867 *: P; MCZ

Holothuria (Selenkothuria) portovallartensis Caso, $1954 * *$ : P

Holothuria (Semperothuria) imitans Ludwig, $1875^{2}$ : P; USNM

Holothuria (Thymiosycia) arenicola Semper, $1868{ }^{5}$. C, P; *Nuevo registro para el Pacífico nicaragüense Holothuria (Thymiosycia) impatiens (Forskål, 1775): C, P; *Nuevo registro para ambas costas de Nicaragua Holothuria (Thymiosycia) thomasi Pawson \& Caycedo, $1980 * *$ : C

Familia Stichopodidae Haeckel, 1896 Astichopus multifidus (Sluiter, 1910) ${ }^{5}$ : C Isostichopus badionotus (Selenka, 1867) ${ }^{5}$ : C Isostichopus fuscus (Ludwig, 1875) 22: P Isostichopus macroparentheses (H.L. Clark, 1922) ${ }^{5}$ : C

Familia Synallactidae Ludwig, 1894 Mesothuria verrilli (Théel, 1886) *: C; TCWC

Orden Elasipodida Théel, 1882

Familia Deimatidae Théel, 1886 Deima validum Théel, 1879 *: C; TCWC

Familia Psychropotidae Théel, 1882

Benthodytes lingua Perrier, 1896 *: C; TCWC Benthodytes typica Théel, 1882 *: C; TCWC Psychropotes depressa (Théel, 1882) *: C; TCWC

Orden Molpadida Haeckel, 1896

Familia Molpadiidae J. Müller, 1850

Molpadia cubana Deichmann, 1940 *: C; TCWC

Orden Apodida Brandt, 1835

Familia Chiridotidae Östergren, 1898 Chiridota rotifera (Pourtalès, 1851): $\mathrm{P}^{2}, \mathrm{C}^{5}$

Familia Synaptidae Burmeister, 1837

Euapta lappa (J. Müller, 1850) ${ }^{5}$ : C

Protankyra cf. ramiurna Heding, 1928 *: C; USNM

Synaptula hydriformis (Lesueur, 1824) ${ }^{1}$ : C; USNM

\section{DISCUSIÓN}

En este trabajo, se suman 56 nuevos registros para Nicaragua, de los cuales 19 corresponden al Pacífico y 38 al Caribe. Llama la atención que muchos de los nuevos registros de equinodermos corresponden a especies comunes en aguas someras del Pacífico y Caribe de México, y de Centroamérica. Tal es el caso de Mithrodia bradleyi, Pharia pyramidata,
Pentaceraster cumingi, Ophiactis savignyi, Ophionereis annulata, Holothuria arenicola, H. impatiens, Hesperocidaris asteriscus, entre otras (Solís-Marín et al., 2013). Lo anterior, podría estar estrechamente relacionado con el esfuerzo de muestreo, ya que, como se muestra en el presente trabajo, se suman más de 50 nuevos registros para el país explorando 10 localidades en cinco días.

Aunque el Pacífico de Nicaragua posee alta diversidad para algunas clases de equinodermos, no existen registros de la clase Crinoidea.

El esfuerzo de muestreo, para el conocimiento de la diversidad de los equinodermos en el Pacífico nicaragüense ha sido mínimo (Quinn-Lampson et al., 2015) por lo cual se propone enfocar futuras investigaciones en esta región. Los estudios realizados en el Caribe, evidencian que existe una alta diversidad de especies de equinodermos para esta región, sin embargo, se debe considerar que fue en el Caribe donde se iniciaron los trabajos de recolección de este grupo, reflejando el esfuerzo de captura en el número de registro de especies.

De las 1539 especies de equinodermos reportadas para América Latina (Alvarado y Solís-Marín, 2013) el número total de especies registradas actualmente para Nicaragua (193), corresponde al 13\%; dicho número y porcentaje están por debajo de países como México, Brasil, Panamá, Cuba, Ecuador, Venezuela, Colombia y Perú, los cuales superan las 200 especies cada uno (Alvarado y Solís-Marín, 2013). Sin embargo, el número de especies de equinodermos de Nicaragua se encuentra por arriba de países como Uruguay, El Salvador, Guatemala, Haití, Honduras, Argentina, República Dominicana, Chile, Puerto Rico, Belice y Costa Rica, donde el número de especies reportado es menor.

El acervo de biodiversidad del grupo en Nicaragua (ejemplares depositados en colecciones científicas) se encuentra fuera del país, especialmente en el United National Museum of Natural History, Smithsonian Institution, Washington, D.C., U.S.A. y en el Museum of Comparative Zoology, Harvard University, U.S.A. 
El inventario de equinodermos de Nicaragua aún dista mucho de estar completo. Se recomienda realizar futuros estudios enfocados a la sistemática molecular, ecología y fisiología de equinodermos de Nicaragua. Es necesario explorar las zonas someras y profundas $(>200 \mathrm{~m})$ con el fin de completar el listado taxonómico de los equinodermos del país. Es muy probable que existan nuevos registros y nuevas especies de equinodermos en ambientes y en regiones poco estudiados de las costas nicaragüenses. El tipo de sustrato, profundidad e inclinación del fondo, entre otros factores, han hecho imposible su muestreo usando métodos convencionales.

El listado taxonómico y los nuevos registros presentados en este trabajo contribuyen al conocimiento de la biodiversidad del país, lo anterior podrá facilitar futuros análisis de vacíos y omisiones en la conservación del grupo, evidenciando sitios de prioridad para su protección.

\section{AGRADECIMIENTOS}

Los autores de este trabajo agradecemos a F. Buitrago-Vannini y E. Castañeda (Escuela de Buceo Dive Nicaragua y ECOLOGICA S.A.) por compartir gentilmente parte de su archivo fotográfico para la inclusión de algunos registros en este trabajo, así como a la Bluefields, Indian and Caribbean University of Corn Island (BICU) por facilitarnos las instalaciones para la realización de trabajo de gabinete. Al Departamento de Biología de la Universidad Nacional Autónoma de Nicaragua (UNAN-León) por facilitarnos parte del equipo requerido en la fase de campo. A A. Durán y a M. E. Diupotex (ICML, UNAM) por las labores técnicas realizadas en el desarrollo de este trabajo.

\section{RESUMEN}

Hay muy poca Información de los Equinodermos de Nicaragua. Aquí nosotros presentamos una lista basada en revisiones bibliográficas y colectas de especímenes hechos en los últimos tres años. El muestreo fue hecho del 11 al 16 de junio de 2016, en 10 localidades (dos en el Pacífico y ocho en el Caribe). Adicionalmente, las bases de datos de ocho colecciones científicas fueron revisadas. Finalmente, se adicionaron registros de archivos fotográficos de la Escuela de Buceo Dive Nicaragua y ECOLÓGICA S.A. La lista de equinodermos de Nicaragua consta de 193 especies distribuidas en cinco clases, 112 géneros, 58 familias y 24 ordenes. La clase con el mayor número de especies fue la clase Asteroidea (57 especies), seguido por la clase Echinoidea (46 especies), y la clase con el menor número de especies fue Crinoidea (10 especies). Cincuenta y seis nuevos registros fueron adicionados para Nicaragua, $19 \mathrm{de}$ los cuales corresponden al Pacífico y 38 al Caribe.

Palabras clave: Echinodermata; Listado actualizado; Corn Island; Little Corn Island; Centro América.

\section{REFERENCIAS}

Alvarado, J.J. (2011). Echinoderm diversity in the Caribbean Sea. Marine Biodiversity, 41, 261-285.

Alvarado, J.J. \& Solis-Marin, F.A. (2013). Echinoderm research and Diversity in Latin America. Berlín: Springer.

Alvarado, J.J., Solís-Marín, F.A., \& C. Ahearn. (2008). Equinodermos (Echinodermata) del Caribe Centroamericano. Revista de Biología Tropical, 3, 37-55.

Alvarado, J.J. Solis-Marin, F. A., \& C. Ahearn. (2010). Echinoderm (Echinodermata) diversity in the Pacific coast of Central America. Marine Biodiversity, 40(1), 45-56.

Alvarado, J.J., Ayala, A., Álvarez del Castillo-Cárdenas, P.A., Fernández, C., Aguirre-Rubí, J., Buitrago, F., \& H. Reyes-Bonilla. (2011). Coral communities of San Juan del Sur, Pacific Nicaragua. Bulletin of Marine Sciences, 87(1), 129-146.

Alvarado, J.J., Barraza, E., \& T.I. Sancho-Mejías. (2013). Central America Echinoderms: Diversity, Ecology and Future Perspectives. In J.J. Alvarado \& F.A. Solís-Marín (Eds.), Echinoderm Research and Diversity in Latin America (pp. 67-99). Berlín: Springer.

Clark, H.L. (1940). Eastern Pacific expeditions of the New York Zoological Society. XXI notes on Echinoderms from the west coast of Central America. Zoologica, 25, 331-352.

Clark, H.L. (1948). A report of the echini of the warmer eastern Pacific, based on the collection of the Velero III. Allan Hancock Pacific Expeditions, 8, 225-352.

Cortés, J. (2007). Coastal morphology and coral reefs: Chapter 7. In J. Bundschuh \& G.E. Alvarado (Eds), Central America: Geology, Resources and Hazards Vol. 1 (pp. 185-200). London: Taylor \& Francis. 
Hendler, G., Miller, J. E., Pawson, D.L., \& Kier, P.M. (1995). Sea Stars, Sea Urchins, and Allies. Echinoderms of Florida and the Caribbean. Washington, D.C.: Smithsonian Institution.

Lyman, T. (1865). Ophiuridae and Astrophytidae. Illustrated Catalogue of the Museum of Comparative Zoology at Harvard College, 1: 1-200.

Quinn-Lampson, S.R., Benito-Sandino, O., Solís-Marín, F.A., Arriaga-Ochoa, J., \& A. Laguarda-Figueras. (2015). Primeros registros de equinodermos (Echinodermata) en aguas someras de Corn Island, Caribe, Nicaragua. Revista de Biología Tropical, 63 (Supl. 2), 27-35.

Ryan, J.D., \& Y. Zapata. (2003). Nicaragua's coral reefs: status, health and management strategies. In J. Cortés (Ed.), Latin American Coral Reefs (pp. 203-222). Amsterdam: Elsevier Science.
Solís-Marín, F.A., Honey-Escandón, M.B.I. HerreroPérezrul, Ma.D., Benítez-Villalobos, F., Díaz-Martínez, J. P., Buitrón-Sánchez, B.E., Palleiro-Nayar J.S., \& A. Durán-González. (2013). Echinoderms from Mexico: Biodiversity, distribution and current state of knowledge. In Alvarado-Barrientos, J.J. \& F.A. SolísMarín (Eds.), Echinoderm research and Diversity in Latin America (pp. 11-65). Berlín: Springer.

Solís-Marín, F.A., Caballero-Ochoa, A.A., Frontana-Uribe, S.C., Laguarda-Figueras, A., \& A. Durán-González. (2017). Catálogo de Autoridades Taxonómicas de los Equinodermos de México. Instituto de Ciencias del Mar y Limnología (ICML), Universidad Nacional Autónoma de México (UNAM). Informe final, SNIB-CONABIO, Proyecto No. Z002. Ciudad de México, México. 